\title{
Trapping of dielectric particles with light-induced space-charge fields
}

\author{
H. A. Eggert, ${ }^{\text {a) }}$ F. Y. Kuhnert, and K. Buse \\ Institute of Physics, University of Bonn, Wegelerstr. 8, D-53115 Bonn, Germany \\ J. R. Adleman \\ Department of Electrical Engineering, California Institute of Technology, Pasadena, California 91125 \\ D. Psaltis \\ School of Engineering, Ecole Polytechnique Fédérale de Lausanne, CH-1015 Lausanne, Switzerland \\ and Department of Electrical Engineering, California Institute of Technology, Pasadena, California 91125
}

(Received 13 April 2007; accepted 18 May 2007; published online 12 June 2007)

\begin{abstract}
Light-induced space-charge fields in lithium niobate crystals are used to trap and manipulate dielectric particles on the surface of such crystals. Without any external voltage source, strong field gradients are present in the proximity of the crystal surface. These are used to trap particles with diameters in the range between $100 \mathrm{~nm}$ and some tens of micrometers. (C) 2007 American Institute of Physics. [DOI: 10.1063/1.2748089]
\end{abstract}

The manipulation of dielectric particles on small length scales is of rising importance in different fields of physics and biomedical research. Optical tweezers are used to manipulate single particles ${ }^{1}$ with high accuracy, but due to the high intensities required, parallel manipulation of many particles is still not feasible. Dielectric particles can be also manipulated electrokinetically, where electric fields provided by electrode patterns are used. ${ }^{2}$ With this method, the particle throughput can be large, but due to the static electrodes the flexibility is low.

Recently, Chiou et al. have reported a method for the manipulation of dielectric particles. ${ }^{3}$ Using optical images on a photoconductive layer, they are able to perform parallel manipulation with up to 15000 traps. Their method still requires an external voltage supply, but the idea can be extended from the usage of photoconductive materials to photorefractive crystals.

Space-charge fields induced in a photorefractive crystal by inhomogeneous illumination ${ }^{4}$ serve as the source of the electric fields outside the crystals. Depending on recording conditions and crystal composition used, the fields inside the crystal can be as strong as $15 \mathrm{kV} / \mathrm{mm}$ (Ref. 5) and the feature sizes are only limited by the light wavelength. Typical recording or erasing times are in the order of seconds or minutes depending on the laser power available. A schematic of the setup is shown in Fig. 1. Arbitrary field patterns can be recorded and fixed either thermally ${ }^{6}$ or optically. ${ }^{7}$

The goal of the present work is to trap particles of sizes between $100 \mathrm{~nm}$ and some tens of micrometers on the surface of a photorefractive crystal. The chemically robust surface of such crystals allows usage also in hostile environments. The strength of this approach is the lack of the need for a voltage supply, as well as its simplicity and robustness.

The dielectrophoretic force $F$ on a spherical dielectric particle in an inhomogeneous electric field is ${ }^{2}$

$$
F=2 \pi r^{3} \epsilon_{m} \frac{\epsilon_{p}-\epsilon_{m}}{\epsilon_{p}+2 \epsilon_{m}} \nabla\left(E^{2}\right),
$$

with the particle diameter $r$, the electric field $E$, and $\epsilon_{m}$ and $\epsilon_{p}$ being the dielectric constants of the surrounding medium

\footnotetext{
${ }^{a)}$ Electronic mail: eggert@physik.uni-bonn.de
}

and the particle, respectively. Since this force depends on the gradient of the square of the electric field, the direction of the field does not matter and, e.g., in a sinusoidal electric field with grating vector $K$, particles will align in a pattern with a grating vector $2 K$, i.e., half of the period length of the fundamental grating. Also, depending on the dielectric constant of the surrounding medium, the particles will either be attracted to $\left(\epsilon_{m}<\epsilon_{p}\right)$ or repelled from $\left(\epsilon_{m}>\epsilon_{p}\right)$ high electric fields.

For the experiments, $4 \mathrm{~mm}$ thick, antireflection coated lithium niobate crystals doped with $0.05 \mathrm{wt} \%$ iron are used. Light of a frequency-doubled $\mathrm{Nd}: \mathrm{YVO}_{4}$ laser is expanded, and the samples are illuminated either with one beam through a binary grating (period length $\Lambda=750 \mu \mathrm{m}$ ) or with two beams that interfere and form a sinusoidal light pattern $(\Lambda=220 \mu \mathrm{m})$. To utilize the strong bulk-photovoltaic effect, the light pattern is always modulated along the $z$ axis of the crystal, and the recording beams are polarized perpendicular to the $z$ axis. In both cases the grating length along the $z$ axis is $5 \mathrm{~mm}$. According to the light pattern, a space-charge field builds up in the crystal. With a light intensity of $600 \mathrm{~mW} / \mathrm{cm}^{2}$ used in the experiments, the saturation field is reached in about $5 \mathrm{~min}$. In the case of a sinusoidal spacecharge field, the field strength in the bulk can be determined by means of light diffraction from the electro-optic refractive-index grating. ${ }^{8}$ For the experiments shown, the amplitude of the space-charge field is about $1.9 \mathrm{kV} / \mathrm{mm}$.

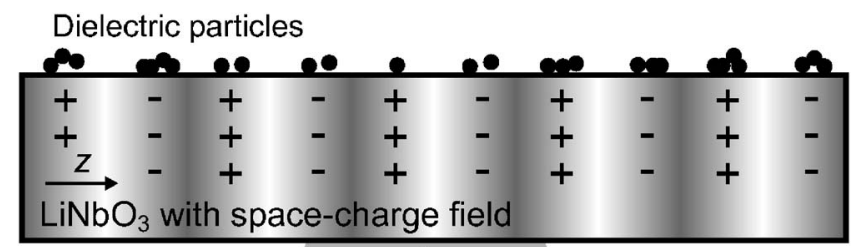

Intensity pattern

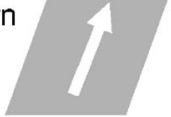

FIG. 1. Schematic of the setup used. A lithium niobate crystal $\left(\mathrm{LiNbO}_{3}\right)$ is illuminated with a light pattern, inducing a space-charge field. This field is used to trap or manipulate dielectric particles on the surface of the crystal. The amplitude of the space-charge field is shown in gray scale and the redistributed charges are denoted + and - . 
(a)
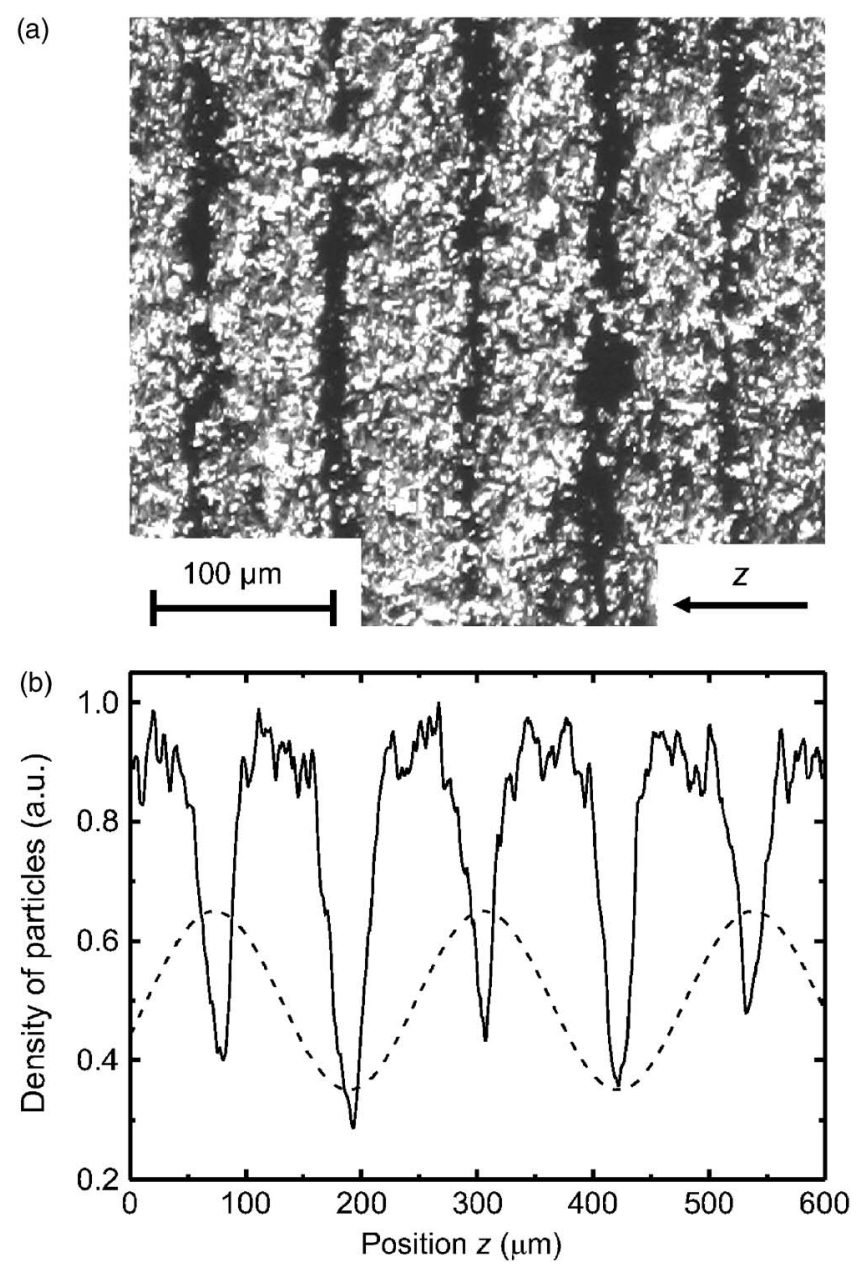

FIG. 2. (a) Photograph of trapped chalk particles on a lithium niobate crystal. A sinusoidal space-charge field with a grating period of $220 \mu \mathrm{m}$ has been recorded inside the crystal, and afterwards the crystal has been homogeneously covered with chalk particles. Then, all not-trapped particles have been blown off with air. (b) Particle concentration vs the $z$ coordinate. It has been obtained by integrating the brightness over the $y$ axis. The dotted line represents the amplitude of the light pattern.

For the experiments in air, chalk particles $\left(\epsilon_{p} \approx 7.5\right.$, diameter of $10-20 \mu \mathrm{m})$ are deposited homogeneously onto one of the $y$ faces of the crystal after recording of the spacecharge field. The delay between completion of the recording and particle deposition ranged between $20 \mathrm{~s}$ and $15 \mathrm{~min}$, which always lead to the same particle distribution. If the particles are brought onto the crystal and the grating is recorded subsequently from the opposite side, a similar distribution is observed. Figure 2(a) shows a reflection-mode microscope photograph of the crystal after excess chalk has been blown off with a low-pressure air flow. The alignment of the particles with a period length of $110 \mu \mathrm{m}$ is in agreement with the $220 \mu \mathrm{m}$ grating period length of the spacecharge field. In Fig. 2(b), the integrated brightness as a function of the $z$ position is shown. Since the chalk is seen as bright spots on the picture, a high brightness represents high particle concentrations. The periodicity of $110 \mu \mathrm{m}$ again is evident, and the characteristics are also in agreement with the expected sinusoidal distribution. One would expect higher and more pronounced maxima, but there is most likely a cutoff because stacked particles cannot be resolved.

With one laser beam direct manipulation of particles is also possible. Illumination of a chalk-covered surface with a Downloaded 12 Nov 2008 to 128.178 .48 .60 . Redistribution subject (a)
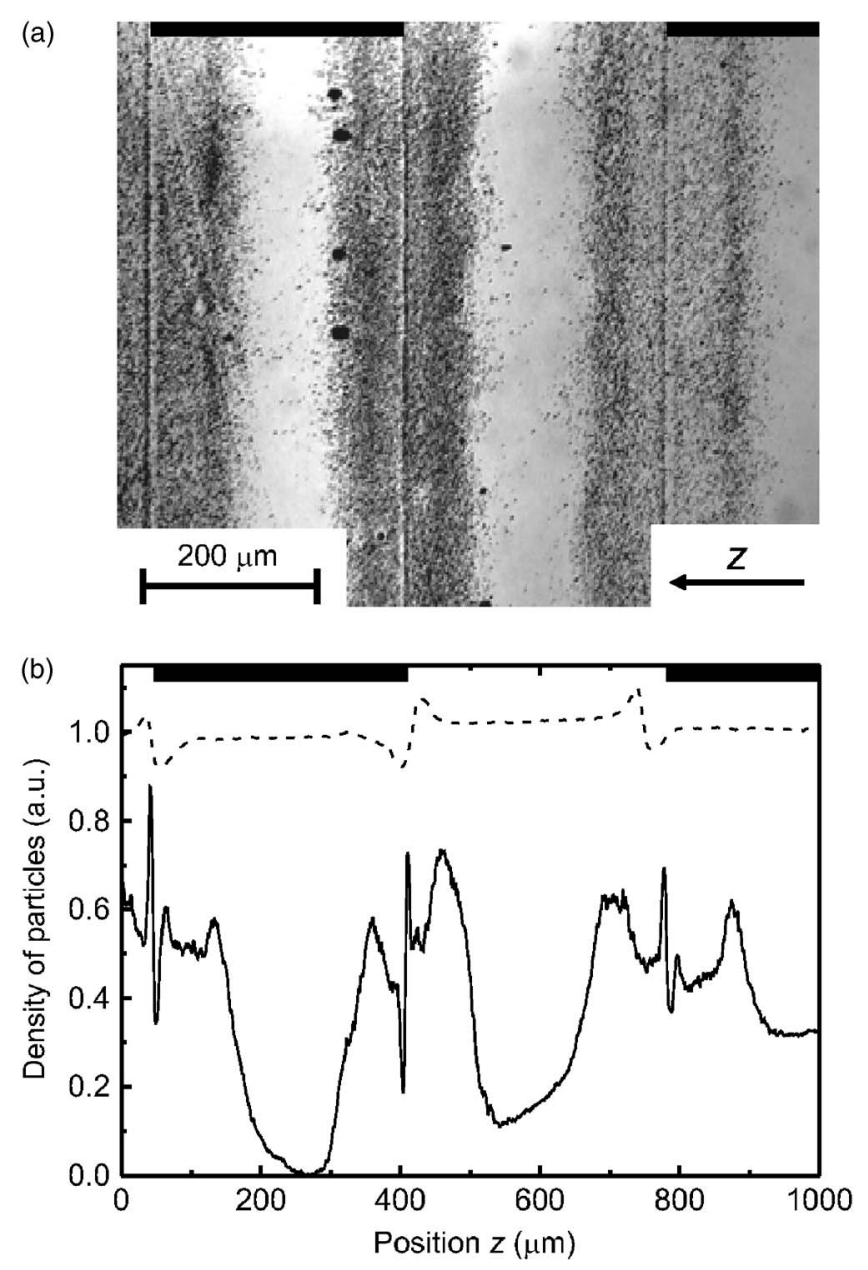

FIG. 3. (a) Trapped silicon carbide ( $\mathrm{SiC})$ particles in paraffin oil on the surface of a lithium niobate crystal. The space-charge field has been recorded with a binary amplitude mask with a grating period of $750 \mu \mathrm{m}$. A drop of oil with the suspended particles has been blown over the grating region. After a few seconds the pattern shown has established itself and is stable for at least $15 \mathrm{~min}$. The $\mathrm{SiC}$ particles appear dark. (b) Particle concentration vs the distance along $z$, obtained by integrating the brightness of (a) along the $y$ axis. The dashed line shows the brightness profile of a photograph of the grating area without particles, taken with differential interference contrast microscopy. In both figures, the dark bars show the position of the grating bars of the binary grating.

Gaussian beam from the back side of the crystal leads to repulsion of the chalk particles out of the illuminated region. For particle steering, the beam has to be moved with a speed slow enough to allow for the buildup of the space-charge field.

Particles can also be trapped in a liquid. A drop of paraffin oil $\left(\epsilon_{m} \approx 1.9\right)$ with silicon carbide particles (diameter of $100 \mathrm{~nm}, \quad \epsilon_{p} \approx 9.7$, volume concentration $\approx 4 \times 10^{-5}$ ) is brought onto the crystal and carefully blown over the grating area. Most of the particles are trapped within approximately $4 \mathrm{~s}$ on the crystal surface. In Fig. 3(a) the resulting particle distribution inside the drop is shown. The periodicity of the particles again is half of the grating period of the spacecharge field. Additionally, a small blank line of about $10 \mu \mathrm{m}$ thickness shows up in the middle of the particle aggregations. The line coincides with the border between dark and illuminated regions during recording, and there is a line of higher particle concentration on the illuminated side, which again is $10 \mu \mathrm{m}$ thick. The particle pattern persists even when paraffin oil flows over it. 
In Fig. 3(b) the particle concentration along the $z$ axis is shown together with the corresponding refractive-index gradients that are obtained by differential interference contrast microscopy. As expected, the particles align on regions with strong changes of the refractive index and hence large field gradients. The trapping regions have a width of about half of the period length of the generated particle grating, and the particle concentration is nearly constant over the area.

One might think that illumination changes the surface chemistry of lithium niobate crystals. To exclude any such influences, crystals have also been illuminated along the $y$ axis with a grating vector normal to the $z$ axis or along the $z$ axis with a grating vector parallel to the $y$ axis. In both cases, no patterns of trapped particles can be seen.

It is possible to use light-induced space-charge gratings to trap particles on the surface of a lithium niobate crystal in air or a liquid. Varying particle distributions arise from the different charge distributions in the bulk of the crystal. The experiments with sinusoidal light patterns (see Fig. 2) show one possible particle distribution induced by the dielectrophoretic force.

A binary intensity pattern leads to strong field gradients at the boundaries between dark and illuminated regions. The actual particle distribution shows unexpected features: The area over which particles are trapped is much broader than it is expected from the refractive-index gradients, and on the border between dark and illuminated regions there is a sharp line where no particles are trapped. The widespread trapping region probably results from particle-particle interaction preventing a higher density by repulsive forces. ${ }^{9}$

The change of the refractive index, i.e., of the electric field at the boundary between dark and illuminated regions is unexpected: In principle positive and negative peaks should be at the borders of the illuminated segments due to charge redistribution by the bulk-photovoltaic effect. The observed structure may originate from a slightly distorted light pattern. In the experiments, the binary grating was placed approximately $120 \mu \mathrm{m}$ above the crystal surface, hence light diffraction may cause the more complicated charge distribution across the boundary regions. The particle distribution across the boundary then is affected by the charge distribution inside the crystal. However, the origin of the force resulting in the thin, sharp line is not clear. Some of the $\mathrm{SiC}$ particles may be charged, hence the distribution can be caused by Coulomb interactions of charged particles and charges inside the crystal. Since more particles always accumulate on the illuminated side of the boundary, all charges carried by particles then need to have the same sign. Also, the dielectro- phoretic force not only has components nearly perpendicular to the surface of the crystal, but, especially close to the charges inside the crystal, it may also have strong components parallel to the surface. This parallel force could be strong enough to push the particles out of a small region and to accumulate them in a line of higher density. Particleparticle interactions can also further influence this distribution.

In conclusion, we have shown the feasibility of using light-induced space-charge fields for trapping of dielectric particles on top of oxidic crystals. The experiments shown open several possibilities: Since the charge transport in photorefractive crystals is well understood, ${ }^{10}$ different field modulations and thus trapping areas can be achieved by using phase and/or amplitude masks or light interference. Using different light patterns, features such as the sharp lines in Fig. 3 can either be prevented or enhanced. Utilizing pulsed illumination and transient gratings, e.g., originating from the pyroelectric effect, ${ }^{11}$ fast changes of the surface field distribution can be achieved. No external voltage source is needed. Therefore, a device can be prepared in the lab and then used for particle manipulation at any place needed. Thermally fixed gratings ${ }^{6}$ together with a simple white-light lamp can also overcome the problem of slow grating decay in the crystals. Photorefractive crystals are furthermore known to have very high spatial resolution, i.e., with masks and ultraviolet light, structures with feature sizes well below $1 \mu \mathrm{m}$ are achievable.

Financial support of the DAAD, the Deutsche Telekom AG, the Deutsche Forschungsgemeinschaft, and the DARPA Center for Optofluidic Integration is gratefully acknowledged. The authors thank Dirk Apitz for helpful and valuable discussions.

\footnotetext{
${ }^{1}$ A. Ashkin, J. M. Dziedzic, and T. Yamane, Nature (London) 330, 769 (1987).

${ }^{2}$ M. P. Hughes, Adv. Colloid Interface Sci. 97, 1 (2002).

${ }^{3}$ P. Y. Chiou, A. T. Ohta, and M. C. Wu, Nature (London) 436, 370 (2005).

${ }^{4}$ Photorefractive Materials and Their Applications I, edited by P. Günter and J.-P. Huignard (Springer, Berlin 1988), Vol. 61.

${ }^{5}$ M. Luennemann, U. Hartwig, and K. Buse, J. Opt. Soc. Am. B 20, 1643 (2003).

${ }^{6}$ J. J. Amodei and D. L. Staebler, Appl. Phys. Lett. 18, 540 (1971).

${ }^{7}$ D. von der Linde, A. M. Glass, and K. F. Rodgers, Appl. Phys. Lett. 25, 155 (1974).

${ }^{8}$ H. Kogelnik, Bell Syst. Tech. J. 48, 2909 (1969).

${ }^{9}$ B. Khusid and A. Acrivos, Phys. Rev. E 54, 5428 (1996).

${ }^{10}$ K. Buse, Appl. Phys. B: Lasers Opt. 64, 391 (1997).

${ }^{11}$ K. Buse, J. Opt. Soc. Am. B 10, 1266 (1993).
} 